\title{
Local Balancing of Low-Voltage Networks by Utilizing Distributed Flexibilities as Part of the InterFlex Field Trial
}

\author{
Nahal Tamadon \\ Thorsten Gross \\ Avacon Netz GmbH \\ Salzgitter, Germany \\ Email: thorsten.gross@avacon.de
}

\author{
Ebrahim Shayesteh \\ KTH Royal Institute of Technology \\ School of Electrical Engineering \\ and Computer Science \\ Stockholm, Sweden
}

\author{
Marco Cupelli, Antonello Monti \\ E.ON Energy Research Center \\ RWTH Aachen University \\ Aachen, Germany
}

\begin{abstract}
The growing share of Distributed Generators (DG) brings with it a fundamental change in dispatchability and location of the overall power generation portfolio. A significant share of DGs are renewable-based and connected to the distribution system, replacing the conventional generators placed at high voltage levels. With this fundamental shift in the power system, more active approaches to distribution system operation are required. In this paper the authors propose a model to utilize distributed flexibilities to locally balance power generation and demand, minimizing the power exchange between low and medium voltage levels. The proposed model is applied to several low voltage networks in the German distribution system. Simulation results show that the proposed model decreases the peak load in tested LV networks by $2.6-12.5 \%$, and leads to an overall flattening of the load profile.
\end{abstract}

\section{INTRODUCTION}

Increasing share of distributed generation in the European power systems has given rise to a number of challenges in the distribution grid, such as voltage deviations, equipment overload, network robustness issues, and reverse power flows $[1,2]$. One way to deal with the challenges of distributed generation and at the same time increase their share is reinforcing the distribution grid, based on a design that allows bidirectional power flows and high DG without encountering voltage instability. Since this approach requires large investments, a great deal of research projects, both academic and industrial $R \& D$, are focused on finding alternative solutions. InterFlex is a project initiated by the European Commission, with the objective to empower distribution system operators (DSOs) in their transition towards more flexible local energy systems. As part of Avacon Netz has defined a use case with the purpose of evaluating the affects of local balancing on the performance of the distribution system.

Local balancing of the distribution system is proposed in [3] as a means to decrease the stress that DG put on the distribution system. That is, [3] proposes an active distribution management approach based on economic dispatch, by using an objective function that minimizes system operation costs, taking into account the voltage and power constraints of the network. The German Renewable Energy Act (EEG), enables distributed RES-based generators to inject power into the grid without being included in an active dispatch mechanism to enhance the integration of RES-based generation. To comply with EEG, and to further promote RES, an alternative approach for local balancing is proposed and evaluated in this paper. The proposed model utilizes the flexibility provided by heat pumps and Electrical Energy Storage (EES) devices owned by residential customers, to increase the load-generation balance at LV level, and thereby decrease power exchange with, and the loading of MV and HV feeders.

Numerous models for utilizing distributed flexibilities have been presented and analyzed in the available literature. The models can be broadly classified into real-time and day-ahead approaches. In a real-time model, the system keeps a regularly updated list of available flexibilities a nd a ctivates $t$ hem as per need, e.g. when voltage or power limits are exceeded as demonstrated in [4], or to match the RES-based generation as demonstrated in [5, 6]. The simulation results in [5] show that in cases where local generation exceeds local load, a real-time approach might result in an overall increase in power consumption. To avoid this, and to ensure optimal resource allocation, a day-ahead approach is adopted here.

For day-ahead scheduling, the majority of the available literature use methods based on optimization. Among these, the most frequent objective functions are cost minimization and profit $\mathrm{m}$ aximization d epending o $\mathrm{n} t$ he $\mathrm{p}$ oint of $\mathrm{v}$ iew of the decision maker, [7-12]. Models for using optimization to improve system performance, e.g. through peak clipping and valley filling [ 13,1 4] o r m inimizing 1 oad variance [15] are also available, but less frequent. A method for increasing PV-power injection in low-voltage networks has been proposed in [16]. The method in [16] however, unlike the method proposed here, is based on hourly price signals, whereas the demand-side management program used here is incentive based, i.e. the market prices do not affect the DSO decisions.

As previously described, the objective of this paper is increasing the local load-generation balance. For this purpose, 
a novel model based on numerical methods is developed. The model creates a day-ahead schedule for distributed flexibilities by allocating the available flexibility resources to the hours with highest load-generation mismatch to decrease the power exchange with the upstream network. The designed algorithm adopt an iterative method for allocating flexibilities to ensure that the flexibility-activation schedule does not cause additional fluctuations in the load profile. The algorithm proposed here has an effect similar to the algorithm proposed in [5]. To the best of the authors' knowledge, local balancing of low-voltage networks using distributed flexibility resources independent of price signals and generation costs are lacking in the literature.

\section{Methodology}

The flexibilities utilized in this use case are electrical heat pumps and EES devices. This section provides a detailed description of the operational principle of these devices, and the algorithm used for local balancing.

\section{A. Electrical heat pumps}

The heat pumps are modeled as in [17], where the indoor temperature at the end of each control period $t$ is given by:

$$
\begin{gathered}
\theta_{t}=\epsilon \theta_{t-1}+(1-\epsilon)\left(\theta_{t}^{A}+\eta_{t}^{C O P} \frac{P_{t}^{H P}}{A}\right) \\
0 \leq P_{t}^{H P} \\
\theta_{\min } \leq \theta_{t} \leq \theta_{\max } \\
\epsilon=\exp [-\Delta t / T C]
\end{gathered}
$$

where

$\theta_{t}\left[{ }^{\circ} \mathrm{C}\right]$ : indoor temperature at the end of period $t$, $\theta_{t}^{A}\left[{ }^{\circ} C\right]$ : ambient temperature during period $t$,

$P_{t}^{H P}[k W]$ : active power consumed by the heater

$\eta_{t}^{C O P}$ : coefficient of performance (COP) of the heater,

$A\left[k W /{ }^{\circ} \mathrm{C}\right]$ : overall thermal conductivity of the building, $\epsilon$ : factor of inertia,

$\Delta t[h]$ : duration of one control period $t$,

$T C$ : time constant of the system.

$\theta_{\min }$ and $\theta_{\max }$ : lower and upper limits of the thermal comfort zone specified in Table II.

\section{B. Electrical Energy Storage}

The operational principle of EES, as defined in $[9,18]$, is given by

$$
\begin{gathered}
E_{t}^{E E S}=E_{t-1}^{E E S}+\eta^{E E S} P_{t}^{E E S} \Delta t \\
E_{\text {min }}^{E E S} \leq E_{t}^{E E S} \leq E_{\max }^{E E S} \\
P_{t}^{E E S} \leq P_{\max }^{E E S} \\
E_{\text {min }}^{E E S}=0.05 E_{r}^{E E S} \quad E_{\text {max }}^{E E S}=0.95 E_{r}^{E E S}
\end{gathered}
$$

where

$E_{t}^{E E S}$ [kWh]: energy stored in the battery at the end of $t$, $\eta^{E E S}$ : charging efficiency of the battery,
$P_{t}^{E E S}[\mathrm{~kW}]:$ power input of the ESS during $t$,

$P_{\max }^{E E S}[\mathrm{~kW}]$ : maximum charging/discharging power,

$E_{\min }^{E E S}$ and $E_{\max }^{E E S}$ recommended lower and upper limits of $E_{t}^{E E S}$ [9]

Depending on whether the EES is being charged or discharged, $P_{k}^{E E S}$ has a positive or negative value respectively.

\section{Scheduling algorithm}

As previously mentioned, the model proposed here creates day-ahead schedules for heat pumps and EES devices, by allocating the flexibility sources to time periods with highest local load-generation mismatch. The day-ahead schedules are created based on load, generation, and temperature forecasts, and perfect forecasting is assumed. The algorithms for EES charging, EES discharging, and heat pump activation are provided in Algorithm 1, Algorithm 2, and Algorithm 3 respectively. The following sections provide a detailed description over the functionalities of each algorithm. These algorithms are described in the same order that they are implemented. The forecasted net load is in each step updated, and passed on to the next algorithm.

1) EES Charging: To increase the local load-generation balance, the algorithm seeks to charge the EES during the time periods when local net load

$$
P^{n e t}=P^{\text {Load }}-P^{D G}
$$

is at its lowest. Here, $P^{\text {Load }}$ and $P^{D G}$ are the local load and generation respectively. Charging all EES capacity in one or two time periods could potentially create fluctuations in the load profile. In order to avoid this, an iterative method is proposed. With this method, during each iteration, the time period with lowest net load is identified and a fraction of the available storage capacity is schedule to be charged during that time period, $t$. The forecasted net load of that time period is updated, taking into account the scheduled EES charging, and a new minimum is identified for allocation of the next fraction of storage capacity. The number of iterations are chosen such that the EES-charging schedule creates as small fluctuations as possible.

The algorithm for EES charging consists of several nested loops. The main loop is the Iteration-accession loop, which creates an empty array to store the indices of the modified time periods, and passes it on to the Capacity-allocation loop. This while loop starts by calculating a fraction of EES capacity, $C_{\text {fraction }}^{E E S}$, to be allocated during each iteration. An array containing the local net-load, $P^{\text {net }}=P^{\text {Load }}+P^{E E S}-P^{D G}$ is then created/updated. The time period with lowest net load, $t$, is then identified and stored in the array containing the modified indices, $\operatorname{Ind}_{\text {modified }}$. The allocated EES capacity, $\triangle E E S$ is chosen such that the following three conditions are met:

- $\triangle E E S \leq C_{\text {fraction }}^{E E S}$

- the EES does not store more than is locally produced

- $P_{t}^{E E S} \leq P_{\max }^{E E S}$ 


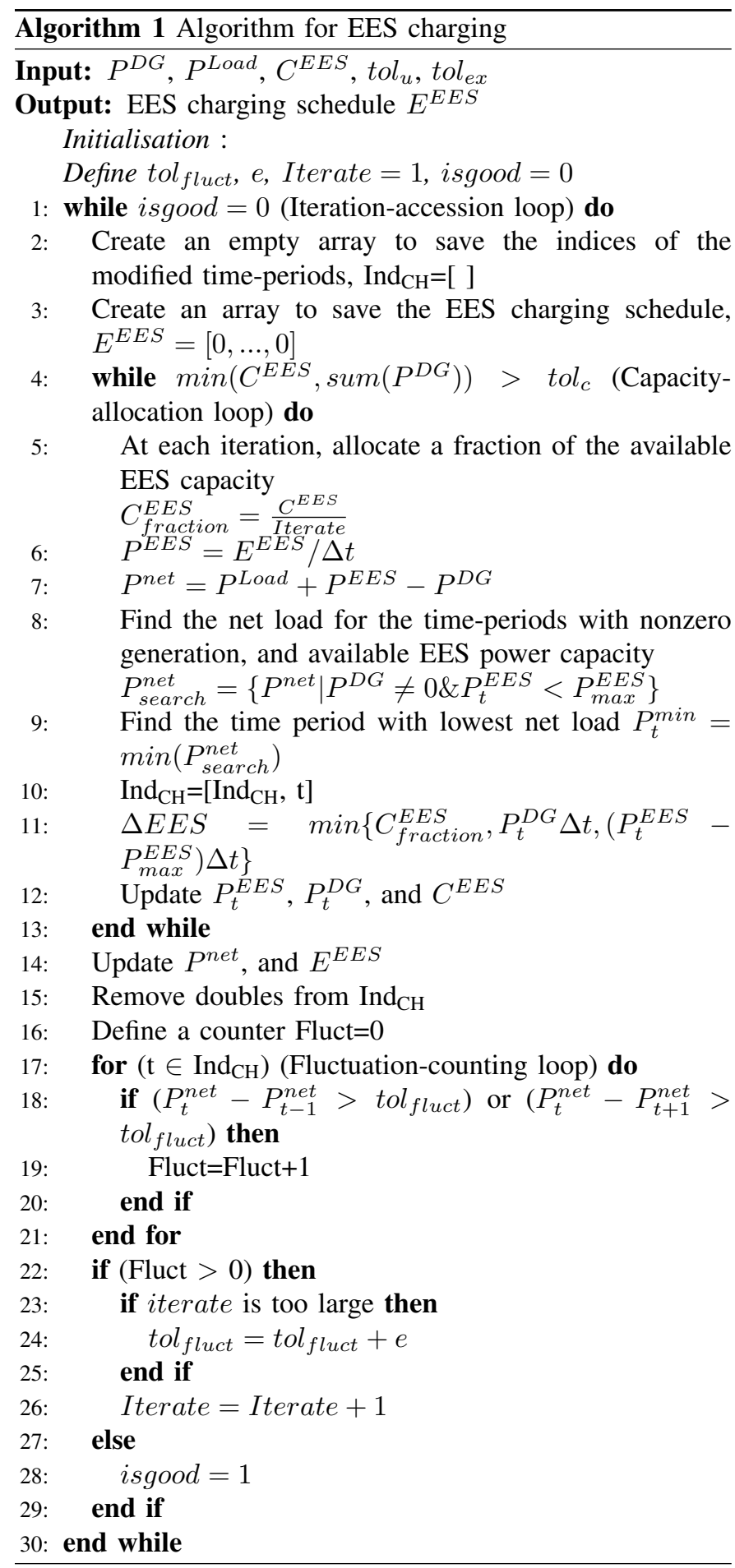

The Capacity-allocation loop then updates $C^{E E S}, P^{D G}$, and $P^{E E S} . P^{D G}$ is updated to make sure that the algorithm does not store more energy than is locally produced and the loop is repeated until either the unallocated part of $C^{E E S}$ or the generated power that has not been stored is smaller than a tolerance value $t o l_{c}$. This value is given by

$$
t o l_{c}=E_{r}^{E E S}-E_{\max }^{E E S}
$$

When the Capacity-allocation loop has finished running, the net load, $P^{\text {net }}$ is updated, taking into account the EES charging schedule. The Fluctuation-counting loop is then used to count the number of fluctuations in $P^{\text {net }}$ created during the modified time periods. To ensure that the algorithm always converges, a tolerance value, $t_{\text {fluct }}$, is used, i.e. if the power fluctuation is less than tol $_{\text {fluct }}$, the fluctuation is neglected. If the EES charging schedule causes power fluctuations, the algorithm checks to see whether the number of iterations has exceeded a preset value, and in that case increases the tolerance for fluctuations. The number of iterations is increased and the Iteration-accession loop is repeated. If the fluctuations caused by EES charging are negligible, the algorithm exits the Iteration-accession loop.

2) EES Discharging: The EES discharging algorithm, similar to the charging algorithm, seeks to increase the local load-generation balance by discharging the EES when local net load, $P^{n e t}$, is at its highest. In order to avoid causing fluctuations in the power profile, this algorithm also adopts an iterative method, during which, a fraction of the available stored capacity is scheduled to be discharged during each iteration. The number of iterations are then successively increased until the obtained discharging schedule causes as small fluctuations as possible.

The discharging algorithm also contains an Iterationaccession loop, which calculates the EES power input, $P^{E E S}$, the total available stored energy, $E^{\text {stored, }}$ and creates an empty array to store the indices of the modified time-periods, Ind $_{m}$ odified. The second main loop is the Energy-allocation loop which starts by calculating a fraction of the stored energy, $E_{\text {fraction }}^{\text {stored }}$, to be allocated during each iteration. The array, $P_{\text {search }}^{\text {net }}$ is then created to limit the search to time periods during which the state of charge of the EES, $S O C^{E E S}$, is nonzero and the discharging power of the EES has not reached its limit. The time-period, $t$, with highest net-load is identified and saved to $\operatorname{Ind}_{D C}$. The amount of stored energy to be allocated, $\triangle E E S$ is then chosen such that

- $\triangle E E S \leq E_{\text {fraction }}^{\text {stored }}$

- the EES does not discharge more than is locally consumed

- $\left|P_{t}^{n e t}\right| \leq P_{\max }^{E E S}$

The Energy-allocation loop then updates $P^{n e t}, P_{t}^{E E S}$, $E^{\text {stored }}$, and $S O C^{E E S}$, and repeats until either the unallocated part of $E^{\text {stored }}$, or the net load is smaller than the tolerance value $t o l_{d c}$. The tolerance is given by

$$
\operatorname{tol}_{d c}=E_{r}^{E E S}-E_{\min }^{E E S}
$$

Similar to the charging algorithm, when the Energyallocation loop has finished running, the Fluctuation-counting loop is then used to count the number of fluctuations in $P^{\text {net }}$ created during the modified time periods. Similarly, a tolerance value, tol $_{\text {fluct }}$, is used to exclude the oscillations that are smaller than tol fluct. If the fluctuations are negligible, the algorithm exits the Iteration-accession loop. Otherwise, the number of iterations is increased and the algorithm is repeated. 


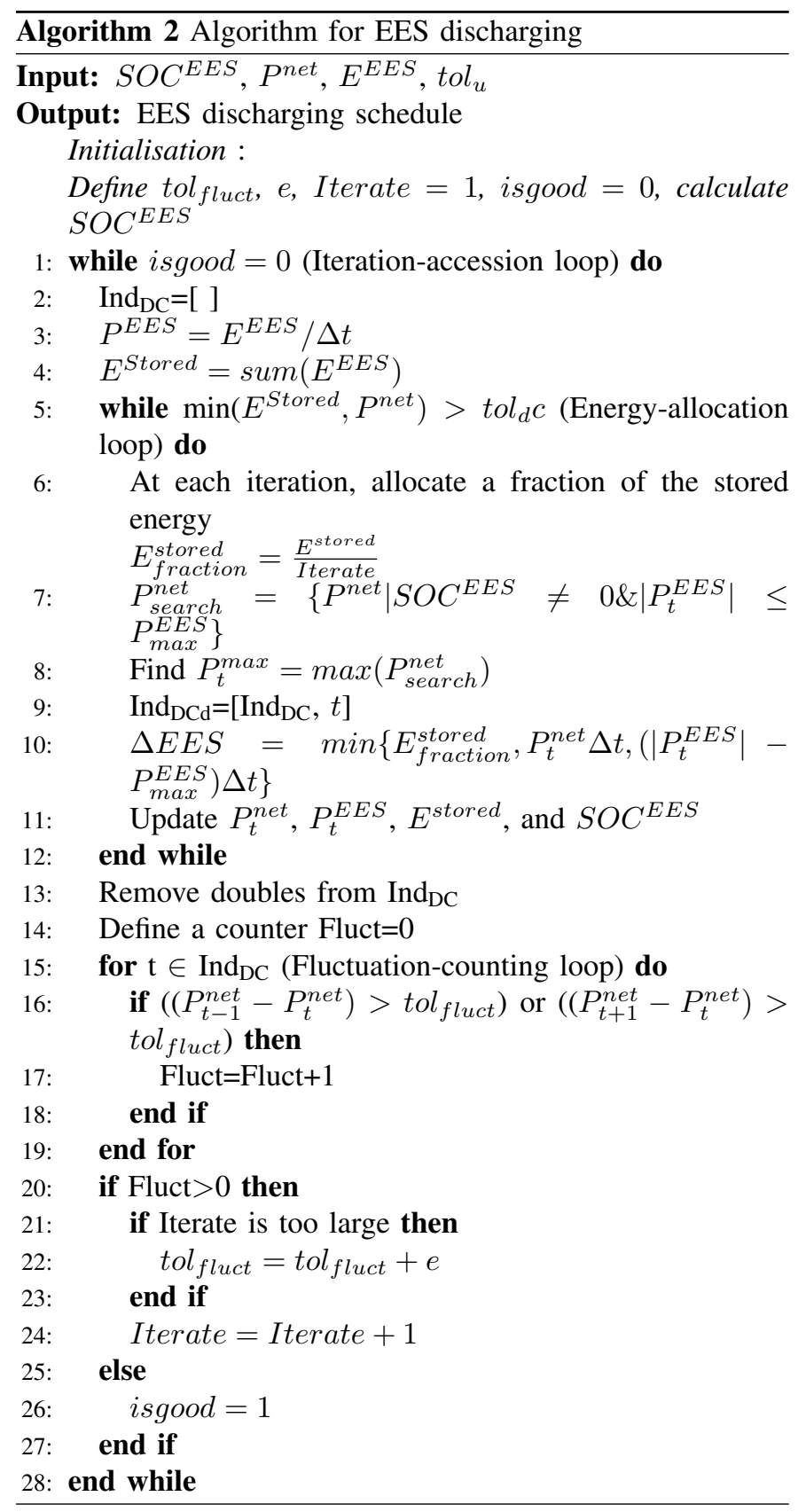

3) Heat-Pump Activation: One of the challenges with thermostatically controlled loads is the load rebound. Assuming that in the absence of DSM, the indoor temperature is kept at the reference temperature $\theta_{\text {ref }}$, if the DSM program decreases the temperature for peak hours, the heating system corrects the low indoor temperature at the end of the peak hours by increasing the heat pump power consumption, causing a rebound. To avoid this, we propose using $P^{H P}$ as the controlled variable, instead of the indoor temperature. For this purpose, three arrays are created:

- $P_{\min }^{H P}$ : the power needed to keep the indoor temperature at $\theta_{\min }$

- $P_{\text {ref }}^{H P}:$ the power needed to keep the indoor temperature
Table I

THERMODYNAMIC PARAMETERS OF BUILDINGS

\begin{tabular}{|llll|}
\hline Parameter & Symbol & Value & Unit \\
\hline System time constant & $T C$ & 25 & $h$ \\
Thermal conductivity & $A$ & 0.14 & $k W /{ }^{\circ} \mathrm{C}$ \\
\hline
\end{tabular}

at $\theta_{\text {ref }}=\frac{\theta_{\min }+\theta_{\max }}{2}$

- $P_{\max }^{H P}:$ the power needed to keep the indoor temperature at $\theta_{\max }$

Using Equation 1, we obtain

$$
P_{\text {ref }}^{H P}=\sum_{t=1}^{T}\left(\frac{A}{\eta_{t}^{C O P}}\right)\left(\theta_{\text {ref }}-\theta_{t}^{A}\right)
$$

$P_{\min }^{H P}$ and $P_{\max }^{H P}$ are obtained by replacing $\theta_{\text {ref }}$ in Equation 12 with $\theta_{\min }$ and $\theta_{\max }$ respectively. Using $P_{\text {ref }}^{H P}$ as the default setting, the algorithm changes $P_{t}^{H P}$ for peak hours to the corresponding values in $P_{\min }^{H P}$, and for the hours with low net load to their corresponding values in $P_{\max }^{H P}$. These hours are selected using $\operatorname{Ind}_{C H}$ and $\operatorname{Ind}_{D C}$ created by the EES charging and discharging algorithms. The heat-pump activation algorithm then calculates the indoor temperature $\theta_{t}$ for each hour using Equation 1, and corrects the heat pump schedule for the time periods when the indoor temperature is outside the comfort zone.

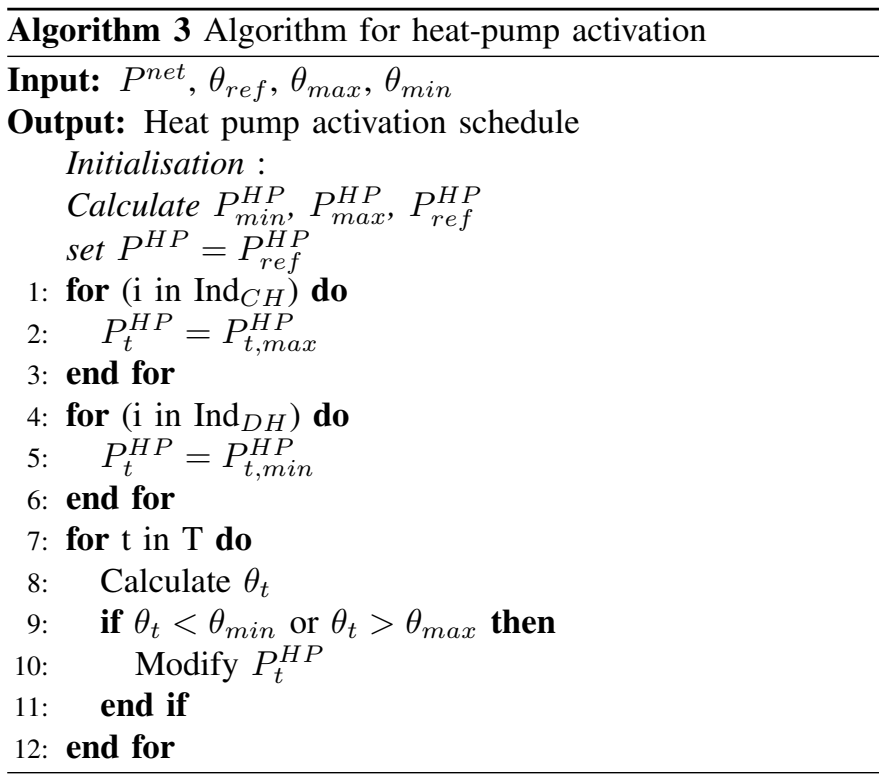

\section{MODEL IMPLEMENTATION}

\section{A. Thermodynamic parameters}

The model used to calculate the indoor temperature as a function of the electricity consumption of the heater is given in Equation 1. Values corresponding to the time constant and the thermal conductivity of a typical building are taken from [17] and presented in Table I.

Table II shows the thermal comfort zones for indoor spaces, specified by the ASHRAE standard [19] Here, we use the 
model proposed in [17] and disregard the humidity.

Table II

THERMAL COMFORT ZONES

\begin{tabular}{|lll|}
\hline & Summer & Winter \\
\hline Indoor Air Temperature & $23-26^{\circ} \mathrm{C}$ & $20-24^{\circ} \mathrm{C}$ \\
\hline
\end{tabular}

The coefficient of performance (COP) is the main factor of interest for evaluating the performance of heat pumps and depends on the temperature difference between the two working environments [20]. The Swedish Energy Agency, Energimyndigheten, performed a series of tests between 2009 and 2013 on 19 different heat pumps [21]. For each heat pump, COP was calculated for four different outdoor temperatures. The mean values of COP at each temperature for these 19 cases are presented in Table III. o obtain the COP for a wider range of temperatures, it is assumed that the polynomial has constant slope between $-20^{\circ} \mathrm{C}$ and $-7^{\circ} \mathrm{C}$, and between $2^{\circ} \mathrm{C}$ and $15^{\circ} \mathrm{C}$. Linear interpolation is then used to acquire $\eta^{C O P}$ for each specific ambient temperature.

Table III

Average COP of Tested Heat Pumps

\begin{tabular}{|lllll|}
\hline $\begin{array}{l}\text { Outdoor } \\
\text { Temperature }\end{array}$ & $-15^{\circ} \mathrm{C}$ & $-7^{\circ} \mathrm{C}$ & $2^{\circ} \mathrm{C}$ & $7^{\circ} \mathrm{C}$ \\
\hline COP & 2.27 & 2.53 & 2.79 & 3.48 \\
\hline
\end{tabular}

\section{B. EES Parameters}

The efficiency of ESS depends on the technology used, and varies between 0.7 and 0.98 [22]. The storage capacity and the rated power of the EES strongly depends on the manufacturing. The technical data of three commercial home battery systems are presented in Table IV. For the simulations in this thesis, the averages of the parameters of these three models are used, presented in the last row of Table IV.

Table IV

EES TECHNICAL DATA

\begin{tabular}{|llll|}
\hline & $\begin{array}{l}E_{r}^{E E S} \\
{[\mathrm{kWh}]}\end{array}$ & $\begin{array}{l}P_{\max }^{E E S} \\
{[\mathrm{~kW}]}\end{array}$ & $\begin{array}{l}\eta^{E E S} \\
{[\%]}\end{array}$ \\
\hline E.ON Aura & 4.4 & 2.5 & 92 \\
Tesla Powerwall & 13.5 & 7 & 90 \\
Sonnenbatterie & 8.0 & 3.3 & 98 \\
Test model & 8.63 & 4.27 & 93.3 \\
\hline
\end{tabular}

\section{Test area}

In order to evaluate the effects of the proposed model, the model is applied to measured grid data of Avacon Netz $\mathrm{GmbH}$. For the simulations, an MV feeder in InterFlex test area is selected. The feeder connects $65 \mathrm{LV}$ networks. The model is applied to eight of these LV networks where the concentration of InterFlex customers is high. Table $\mathrm{V}$ provides an overview of the Installed PV capacity, number of household connections, and number of heat pumps and EES owned by InterFlex customers.
Table V

INTERFLEX LOW-VOLTAGE NETWORKS

\begin{tabular}{|lllllll|}
\hline $\begin{array}{l}\text { Bus } \\
\text { number }\end{array}$ & PV & \multicolumn{2}{c}{ Household } & \multicolumn{2}{c}{ Heat Pumps } & \multicolumn{2}{c|}{ EES } \\
\hline 1 & {$[\mathrm{MW}]$} & connections & $\begin{array}{l}\text { Case } \\
\text { A }\end{array}$ & Case B & Case & A \\
2 & 0.011 & 125 & 2 & 38 & 1 & 3 \\
3 & 0.08 & 105 & 3 & 28 & 1 & 6 \\
4 & 0.026 & 104 & 2 & 31 & 1 & 4 \\
5 & 0.044 & 93 & 2 & 21 & 1 & 6 \\
6 & 0.153 & 59 & 1 & 13 & 1 & 3 \\
7 & 0.080 & 54 & 4 & 17 & 2 & 2 \\
8 & 0.070 & 187 & 3 & 52 & 1 & 13 \\
\hline
\end{tabular}

\section{Simulation cases}

The system is simulated for three different cases, as described below:

- Base Case: this corresponds to the status quo of the system

- Case A: the proposed model is applied to the devices that are currently available to InterFlex

- Case B: based on an estimation performed by Avacon Netz, the number of heat pumps available to InterFlex corresponds to $8 \%$ of the total number of residential heat pumps in the test area. For Case B, it is assumed that all PV generators are equipped with EES and the proposed model is applied to all the heat pumps and EES devices available in the area. To estimate the total number of heat pumps in each LV network, i.e. to go from the approximate $8 \%$ utilized in Case A to utilizing 100\% of the heat pumps, the number of heat pumps in each network is scaled with a factor of 12.5. The number of heat pumps is then distributed among LV networks with respect to the number of residential customers in each network.

For all three cases, simulations are performed for the following two days:

- Day 1: the day with highest PV generation. This occurred on a weekday in summer

- Day 2: a weekday in winter

Each simulation is performed over a $24 \mathrm{~h}$ period with 15 minute intervals:

$$
\Delta t=15 \text { minutes } \quad T=96
$$

To evaluate the collective affect of the peak reductions, the active power loading of the MV feeder is also analyzed for the different cases. For this purpose we also look at the load factor of the MV feeder, given by [23]

$$
\text { Load } \quad \text { Factor }=\frac{\text { Average Load }}{\text { Peak Load }}
$$

\section{RESUlTS}

\section{A. Base Case, status quo}

As previously mentioned, eight LV networks are used for the simulations in this study. Figures 1 and 2 show the net load for each network during Day 1 and 2. The load of each network 
includes the residential load and the total power consumption of the heat pumps in the area. It can be seen that during the day with highest PV generation, the net load in bus 5 is negative, i.e. this network might cause reverse power flows on days with high PV generation. The effect of the model on the reverse power flow in Bus 5 is analyzed in Section IV-E. The load variations are clear in all eight networks, with distinct evening peaks.

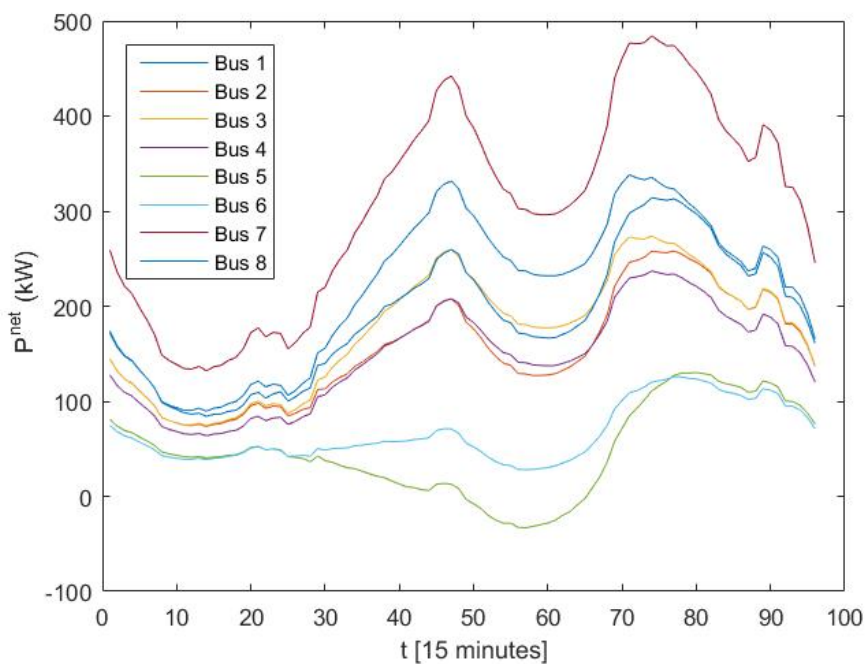

Figure 1. Net Load of InterFlex Buses, Day 1 (summer)

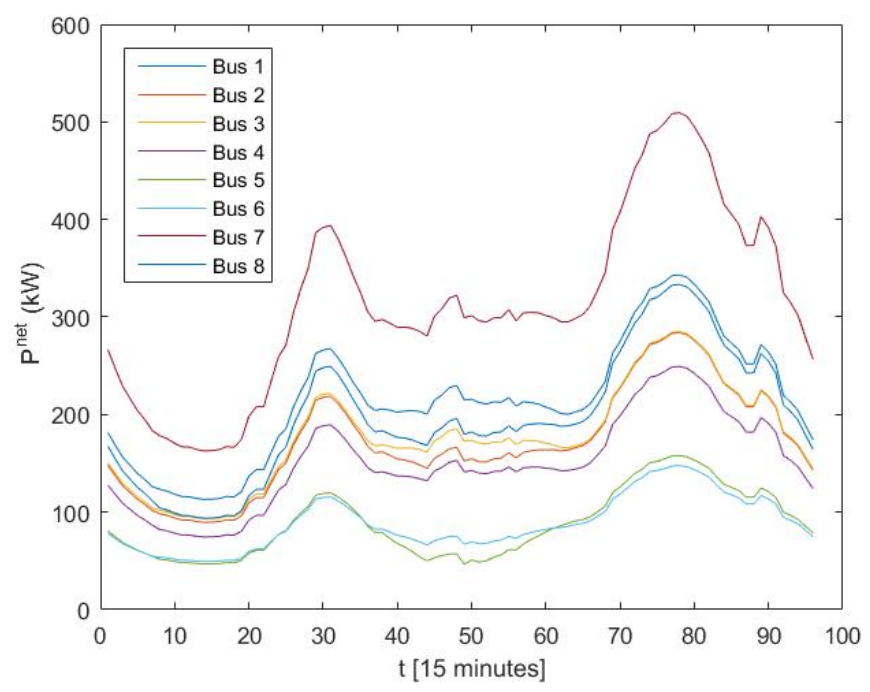

Figure 2. Net Load of InterFlex Buses, Day 2 (winter)

\section{B. Case A, InterFlex flexibilities}

The proposed model is applied to the buses in Table V. Table VI shows the reduction in peak load for each bus caused by the heat pump and EES schedules both in [kW] and as a percentage. It can be seen that the proposed model reduces the peak load of each network with $6.98-12.37 \mathrm{~kW}$, alternatively by $2.43-8.51 \%$.
Table VI

Peak Reduction in Case A

\begin{tabular}{|lllll|}
\hline $\begin{array}{l}\text { Bus } \\
\text { number }\end{array}$ & {$[\mathrm{kW}]$} & {$[\%]$} & {$[\mathrm{kW}]$} & {$[\%]$} \\
\hline 1 & 9.83 & 2.91 & 10.76 & 3.13 \\
2 & 7.33 & 2.84 & 10.13 & 3.57 \\
3 & 7.76 & 2.83 & 10.08 & 3.54 \\
4 & 8.54 & 3.60 & 9.71 & 3.89 \\
5 & 6.98 & 5.36 & 8.02 & 5.08 \\
6 & 10.71 & 8.51 & 11.36 & 7.70 \\
7 & 11.94 & 2.46 & 12.37 & 2.43 \\
8 & 8.66 & 2.76 & 10.80 & 3.24 \\
\hline
\end{tabular}

Table VII

PEAK Reduction In CASE B

\begin{tabular}{|lllll|}
\hline $\begin{array}{l}\text { Bus } \\
\text { number }\end{array}$ & {$[\mathrm{kW}]$} & {$[\%]$} & {$[\mathrm{kW}]$} & {$[\%]$} \\
\hline 1 & 17.66 & 5.22 & 17.98 & 5.24 \\
2 & 25.90 & 10.03 & 31.87 & 11.23 \\
3 & 19.29 & 7.05 & 23.08 & 8.10 \\
4 & 26.10 & 11.01 & 30.76 & 12.34 \\
5 & 14.95 & 11.48 & 16.88 & 10.69 \\
6 & 10.81 & 8.60 & 11.88 & 8.05 \\
7 & 54.15 & 11.18 & 56.83 & 11.16 \\
8 & 28.24 & 9.00 & 34.48 & 10.34 \\
\hline
\end{tabular}

\section{Case B, potential flexibilities}

For the simulations in Case B, all the available heat pumps and EES devices in the test area are utilized. The reductions in peak load are demonstrated in Table VII in $k W$, and as a percentage of original peak load. It can be seen that the peak reductions in Case B vary between 10.81-56.83 kW, and 5.22-12.34\%.

\section{Loading of the medium-voltage feeder}

Figures 3 and 4 show the active power loading of the MV feeder that connects the InterFlex buses. For the analysis in this case, it is assumed that the InterFlex buses are the only buses connected to the feeder. It can be seen that the proposed model has a peak shaving and valley filling effect, resulting in an overall flattening of the load profile. It can also be seen that for both days, the effects in Case B are more substantial, which was anticipated since the number of flexible devices is higher in Case B compared to Case A.

An overview of the effects of local balancing on the MV feeder is provided in Table VIII. The load factor is obtained as specified by Equation 13. Note that the peak reduction for the MV feeder does not equal the sum of all peak reductions in the LV networks, because the net load in the LV networks do not reach their peak values simultaneously. 


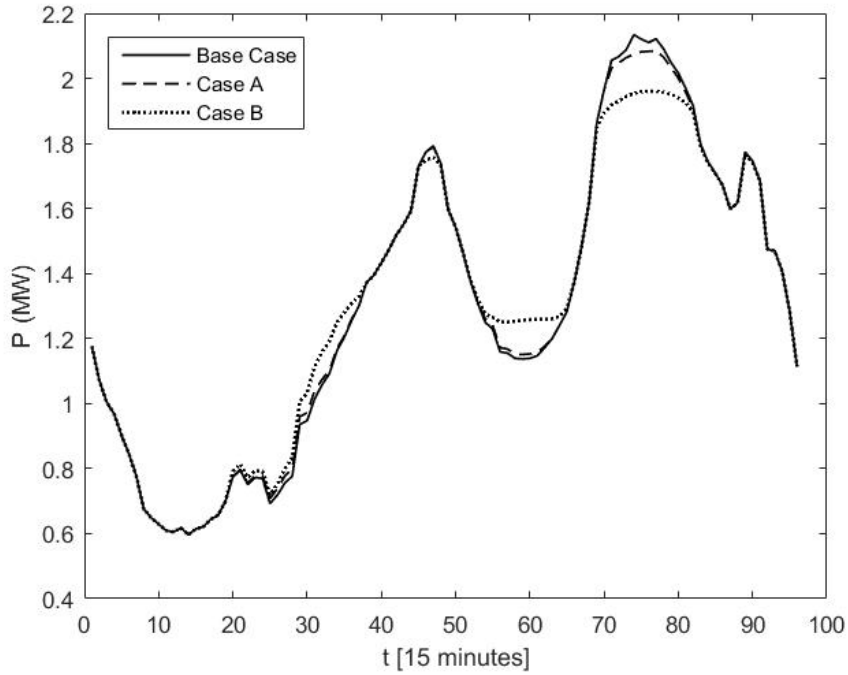

Figure 3. Active Power Loading of the Medium-Voltage Feeder Day 1 (summer)

Table IX

REVERSE POWER IN BUS 5, DAY 1

\begin{tabular}{|llll|}
\hline & Peak $[\mathrm{kW}]$ & Sum $[\mathrm{kW}]$ & $\begin{array}{l}\text { Duration } \\
{[\text { minutes }]}\end{array}$ \\
\hline Base Case & 33.08 & 353.31 & 255 \\
Case A & 28.76 & 317.63 & 255 \\
Case B & 19.54 & 239.35 & 255 \\
\hline
\end{tabular}

Table VIII

Peak Reduction And LoAd Factor of the MV FeEder

\begin{tabular}{|lllll|}
\hline & & \multicolumn{3}{c|}{ Peak Reduction } \\
& & {$[\mathrm{kW}]$} & {$[\%]$} & Load Factor \\
\hline \multirow{2}{*}{ Day 1 } & Base Case & & & 0.61 \\
& Case A & 48.92 & 2.29 & 0.63 \\
& Case B & 173.47 & 8.13 & 0.67 \\
\hline \multirow{2}{*}{ Day 2 } & Base Case & & & 0.61 \\
& Case A & 83.81 & 3.63 & 0.63 \\
& Case B & 230.71 & 9.99 & 0.68 \\
\hline
\end{tabular}

\section{E. Reverse power flow}

As it was demonstrated in Figure 1, the PV generation in Bus 5 causes reverse power flows on Day 1. Figure 5 shows Pnet for Bus 5 during Day 1, for all three cases.

As it can be seen in the Figure, the proposed model decreases both the reverse power flow and the peak load of Bus 5 . The values of the reverse power flow are provided in more detail in Table IX. The results show that the model decreases the peak reverse power with $13.06 \%$ in Case A and $40.93 \%$ in Case B. The model also reduces the total amount of reverse power by $10.10 \%$ and $32.25 \%$ in Case A and B respectively. The model however, does not affect the duration of reverse power flow.

\section{CONCLUSION}

In this paper, a model for day-ahead scheduling of heat pumps and EES based on numerical methods was proposed.

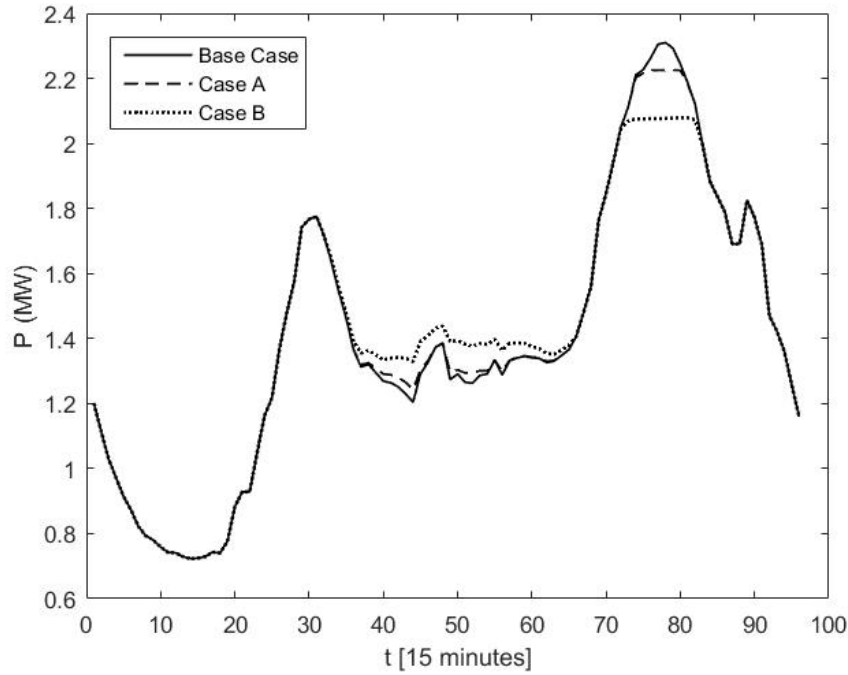

Figure 4. Active Power Loading of the Medium-Voltage Feeder, Day 2 (winter)

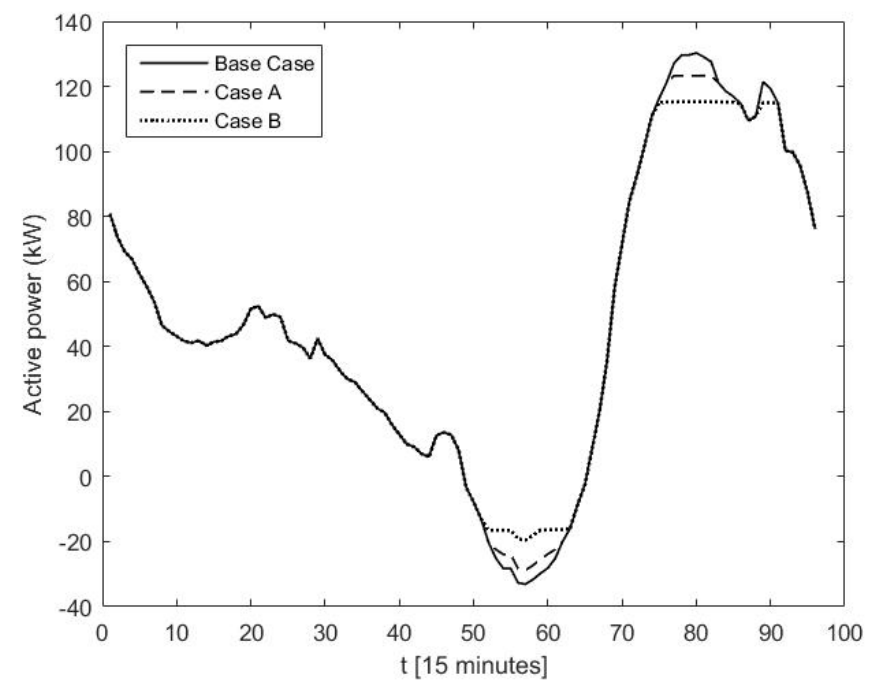

Figure 5. Net Load of Bus 5, Day 1 (summer)

The objective of the model is locally balancing the LV networks and thereby decreasing the loading of higher-voltage equipment. The proposed model was applied to eight LV networks in the InterFlex test area, based on measured grid data of Avacon Netz GmbH. Two sets of simulations were performed: Case A considering the devices available to InterFlex, and Case B taking into account all the heat pumps and EES in said area. The model proved effective in reducing the peak load of LV networks, resulting in an overall decrease of the loading on the MV feeder connecting these LV networks. The model also showed promising results on decreasing the load factor of the MV feeder, and in reducing reverse power flows caused by high local generation.

Potential future work includes sensitivity analysis to eval- 
uate the effects of the different parameters such as local generation, number of heat pumps and EES capacity on the model. An economical analysis can also be performed to evaluate the effects of the model on the operational and capital expenditure of distribution system operation.

\section{ACKNOWLEDGMENT}

We wish to acknowledge the contributions of all experts involved in the project InterFlex (Grant No: 731289) co-funded by the European Commission as part of the H2020 Program (H2020-LCE-2016-SGS). Website: http://interflex-h2020.com/

\section{REFERENCES}

[1] H. Ahmadi, A. Ellis, and J. R. Marti, "Voltage management challenges in feeders with high penetration of distributed generation," in 2016 IEEE Canadian Conference on Electrical and Computer Engineering (CCECE), pp. 1-6, May 2016.

[2] R. Belmans and A. I. Ramos Gutierrez, "Coordination of flexibility contracting in wholesale and local electricity markets ; coördinatie voor het verhandelen van flexibiliteit in groothandel en in lokale elektriciteitsmarkten ,," June 2017.

[3] M. Wierzbowski and B. Olek, "Market-based local balancing in distribution networks," in IET Conference Proceedings, (Stevenage), The Institution of Engineering \& Technology, October 2013.

[4] C. Vivekananthan, Y. Mishra, G. Ledwich, and F. Li, "Demand response for residential appliances via customer reward scheme," IEEE Transactions on Smart Grid, vol. 5, pp. 809-820, March 2014.

[5] L. Zhang, N. Chapman, N. Good, and P. Mancarella, "Exploiting electric heat pump flexibility for renewable generation matching," pp. 1-6, IEEE, June 2017.

[6] M. Liu, F. L. Quilumba, and W. J. Lee, “A collaborative design of aggregated residential appliances and renewable energy for demand response participation," IEEE Transactions on Industry Applications, vol. 51, pp. 35613569, Sept 2015.

[7] P. Hasanpor Divshali, B. J. Choi, H. Liang, and L. Söder, "Transactive demand side management programs in smart grids with high penetration of EVs," Energies, vol. 10, no. 10, 2017.

[8] M. Alham, M. Elshahed, D. K. Ibrahim, and E. E. D. A. El Zahab, "Optimal operation of power system incorporating wind energy with demand side management," Ain Shams Engineering Journal, vol. 8, pp. 1-7, March 2017.

[9] W. Lee, J. Jung, and M. Lee, "Development of 24-hour optimal scheduling algorithm for energy storage system using load forecasting and renewable energy forecasting," in 2017 IEEE Power Energy Society General Meeting, pp. 1-5, July 2017.

[10] H. Khani and H. E. Z. Farag, "Optimal scheduling of energy storage to mitigate power quality issues in power systems," in 2017 IEEE Power Energy Society General Meeting, pp. 1-5, July 2017.
[11] S. Choi and S. W. Min, "Optimal scheduling and operation of the ess for prosumer market environment in grid-connected industrial complex," IEEE Transactions on Industry Applications, vol. 54, pp. 1949-1957, May 2018.

[12] S. Gottwalt, J. Gärttner, H. Schmeck, and C. Weinhardt, "Modeling and valuation of residential demand flexibility for renewable energy integration," IEEE Transactions on Smart Grid, vol. 8, pp. 2565-2574, Nov 2017.

[13] P. Balakumar and S. Sathiya, "Demand side management in smart grid using load shifting technique," in 2017 IEEE International Conference on Electrical, Instrumentation and Communication Engineering (ICEICE), pp. 16, April 2017.

[14] T. Logenthiran, D. Srinivasan, and T. Z. Shun, "Demand side management in smart grid using heuristic optimization," IEEE Transactions on Smart Grid, vol. 3, pp. 1244-1252, Sept 2012.

[15] O. Erdinc, N. G. Paterakis, T. D. P. Mendes, A. G. Bakirtzis, and J. P. S. Catalão, "Smart household operation considering bi-directional ev and ess utilization by real-time pricing-based dr," IEEE Transactions on Smart Grid, vol. 6, pp. 1281-1291, May 2015.

[16] N. Karthikeyan, B. R. Pokhrel, J. R. Pillai, B. BakJensen, and K. H. B. Frederiksen, "Demand response in low voltage distribution networks with high PV penetration," in 2017 52nd International Universities Power Engineering Conference (UPEC), pp. 1-6, Aug 2017.

[17] P. Constantopoulos, F. Schweppe, and R. Larson, "Estia: A real-time consumer control scheme for space conditioning usage under spot electricity pricing," Computers and Operations Research, vol. 18, no. 8, pp. 751-765, 1991.

[18] A. A. Thatte and L. Xie, "Towards a unified operational value index of energy storage in smart grid environment," IEEE Transactions on Smart Grid, vol. 3, pp. 1418-1426, Sept 2012.

[19] M. d. M. Castilla, Comfort Control in Buildings. Advances in Industrial Control, 2014.

[20] W. Grassi, Heat Pumps Fundamentals and Applications. Green Energy and Technology, 2018.

[21] "Test results - air/air heat pumps (luftvärmepumpar)," Swedish Energy Agency, 2009-2013. [Online; accessed 12-April-2018] Available: http://www.energimyndigheten.se/tester/testera-o/luftluftvarmepumpar-20092013/?productTypeVersionId=1392.

[22] A. Alhamali, M. E. Farrag, G. Bevan, and D. M. Hepburn, "Review of energy storage systems in electric grid and their potential in distribution networks," pp. 546551, IEEE, December 2016.

[23] F. C. Robert, G. S. Sisodia, and S. Gopalan, "The critical role of anchor customers in rural microgrids: Impact of load factor on energy cost," in 2017 International Conference on Computation of Power, Energy Information and Commuincation (ICCPEIC), pp. 398-403, March 2017. 